\title{
FATOQRES ASȘOCIADOS À NÃO ADESÃO ÀS POLIITICAS PÚBLICAS DE USO E VENDA DO ÁLCOOL NO BRASIL: I LEVANTAMENTO NACIONAL DE ÁLCOOL E DROGAS
}

\section{FACTORS ASSOCIATED WITH NON-ADHERENCE TO PUBLIC POLICIES RELATED TO ALCOHOL USE AND SALE IN BRAZIL: FIRST NATIONAL SURVEY ON ALCOHOL AND DRUGS}

\section{Resumo}

Este estudo buscou analisar, em uma amostra representativa da população brasileira, as prevalências de aceitação de políticas relacionadas ao acesso, promoção, prevenção e tratamento dos problemas relacionados ao uso do álcool, bem como sua anuência para possíveis mudanças. Investigou, ainda, o perfil dos indivíduos não favoráveis às políticas por meio de avaliação da associação com variáveis sociodemográficas, consumo de outras substâncias psicotrópicas, acesso a campanhas de prevenção e exposição a propagandas de bebidas. Trata-se de estudo com desenho transversal usando dados do I Levantamento Nacional de Álcool e Drogas. Uma amostra de 3.007 participantes responderam escalas sobre 16 políticas restritivas. Uma escala de aceitação foi criada, e o grupo dos $5 \%$ que menos aderiam às políticas foi analisado. As associações foram realizadas através de modelos ajustados de regressão logística (StataSE10). Observou-se que a maioria da população investigada apoia as políticas vigentes, assim como a implementação de novas leis de restrição ao consumo de álcool. Os fatores preditores de não aceitação das políticas foram: ser homem, jovem, sem relacionamento estável, com maior escolaridade, não religioso e tabagista. O uso substâncias ilícitas e abuso e/ou dependência de álcool também foram associados a não adesão às políticas de restrição avaliadas. As políticas referindo tratamento para 0 alcoolismo e restrição de propagandas foram as mais aceitas.
Incentiva-se iniciativas de esclarecimento da importância das políticas de saúde pública direcionadas ao perfil do público menos adepto.

Palavras-chave: Políticas públicas, álcool, prevenção.

\section{Abstract}

This study aimed to examine the prevalence of public acceptance of several restriction policies related to alcohol use, sale, and advertising in the Brazilian population. It also investigated factors associated with non-adherence to those policies. This cross-sectional study used data from the First Brazilian National Survey on Alcohol and Drugs. A sample of 3,007 participants completed scales regarding 16 restriction policies. An acceptance scale was created, and the top 5\% of respondents showing the lowest acceptance rates were analyzed. Associations were assessed using adjusted logistic regression models (StataSE10). Most respondents were favorable to the restriction policies presented and to the implementation of new policies to further restrict alcohol consumption. The factors predicting non-adherence to the policies were: being male, young, single, well educated, not religious, and smoker. The use of illegal substances and alcohol abuse/dependence were also associated with non-adherence to the restriction policies. Policies related to treatment for alcohol dependence and alcohol advertising restrictions were the ones most commonly accepted. Future actions are 


\section{CAROLINA DE MENESES GAYA ${ }^{1}$, CLARICE SANDI MADRUGA², ANDRÉ DE QUEIROZ CONSTANTINO MIGUEL ${ }^{2}$, SANDRO MITSUHIRO², ILANA PINSKY², RAUL CAETANO ${ }^{3}$, \\ RONALDO LARANJEIRA ${ }^{2}$}

${ }^{1}$ Instituto Nacional de Ciência e Tecnologia para Políticas Públicas do Álcool e Outras Drogas, Instituto de Psiquiatria, Universidade Federal de São Paulo (UNIFESP), São Paulo, SP. Universidade de Franca, Franca, SP. ${ }^{2}$ Universidade de Franca, Franca, SP. ${ }^{3}$ University of Texas School of Public Health, Dallas Regional Campus, Dallas, TX, EUA.

warranted to inform the population less susceptible to adhere of the importance of public health policies.

Keywords: Public policies, alcohol, prevention.

\section{INTRODUÇÃo}

Entre todos os países em desenvolvimento, o Brasil apresenta um dos maiores índices de problemas relacionados ao consumo de álcooll-3. Esses problemas tendem a piorar com o crescimento do país, uma vez que há uma relação direta entre prosperidade econômica e consumo de álcool nos países em desenvolvimento ${ }^{1,4,5}$.

Grandes corporações são capazes de manipular estilos de vida, promovendo produtos, criando desejos e influenciando decisões. No Brasil, a indústria do álcool enfrenta poucos obstáculos em relação às políticas públicas. Até pouco tempo atrás, não existiam leis restringindo a venda de produtos alcoólicos nas margens das principais estradas do país. Não adotamos a política de "licença para venda de bebidas", usada na maioria dos países desenvolvidos, o que leva a uma ampla e indiscriminada rede de venda do produto. Tal amplitude de acesso dificulta a fiscalização e o controle, o que aumenta significativamente o consumo entre a população 2,6,7.

Entende-se por políticas públicas de restrição ao uso de álcool um conjunto de medidas desenvolvidas pelo governo ou por grupos não governamentais para minimizar ou prevenir os problemas relacionados ao consumo de bebidas alcoólicas ${ }^{8}$. Essas políticas podem envolver a implementação de estratégias específicas, como, por exemplo, restrições ao consumo de álcool por menores e alocação de recursos para ações preventivas ou de tratamento. Entre as inúmeras estratégias e intervenções recomendadas pela Organização Mundial de Saúde (OMS), destacam-se: aumento do preço das bebidas, regulação da disponibilidade física do álcool, maior fiscalização do beber e dirigir, regulação da promoção de bebidas alcoólicas, estratégias educacionais, tratamento e intervenções breves ${ }^{9-11}$.

Convém ressaltar que a compreensão e o apoio da população são elementos imprescindíveis para o planejamento, direcionamento e implementação de políticas públicas sobre o uso de álcool. Além da adesão da opinião pública, essas políticas devem ser baseadas em evidências científicas e em consensos entre especialistas e autoridades.

$\bigcirc$ presente estudo analisou a opinião de uma amostra representativa da população brasileira sobre as políticas públicas existentes relacionadas ao acesso, promoção, prevenção e tratamento dos problemas relacionados ao uso do álcool, bem como sua anuência para possíveis mudanças. Investigou, ainda, o perfil dos indivíduos não favoráveis às políticas por meio de avaliação da associação com variáveis sociodemográficas, consumo de outras substâncias psicotrópicas, acesso a campanhas de prevenção e exposição a propagandas de bebidas.

\section{Material e métodos}

\section{Amostragem e procedimentos}

A primeira onda do Levantamento Nacional de Álcool e Drogas (I LENAD) foi realizada em 2006. O método de amostra probabilística estratificada em múltiplos estágios foi utilizado para selecionar 3.007 indivíduos maiores de 14 anos na população da brasileira (taxa de resposta $=66,4 \%$ ). A amostragem envolveu três estágios: 1) seleção de 143 municípios pelo método de probabilidade proporcional ao tamanho (PPS), com estratificação dos municípios por região administrativa e porte; 2) seleção de dois setores censitários para cada município, com exceção dos 14 maiores municípios selecionados, totalizando 325 setores censitários, utilizando também PPS; e 3) dentro de cada setor censitário, oito domicílios foram selecionados por amostragem aleatória simples, seguida da seleção de um membro da família a ser entrevistado usando a técnica do "mais próximo do futuro aniversário". Entrevistas individuais face a face foram realizadas na casa do entrevistado por entrevistadores treinados por meio de um questionário padronizado com duração média de 53 minutos.

\section{Medidas}

Utilizou-se uma versão adaptada para a população brasileira do questionário Hispanic Americans Baseline Alcohol Survey $(\mathrm{HABLAS})^{12}$ para avaliar as medidas sociodemográficas e os padrões de consumo de álcool, tabaco e drogas ilícitas. Abuso e dependência de álcool foram definidos de acordo com a $4^{a}$ edição do Manual Diagnóstico e Estatístico de Transtornos Mentais (DSM-IV). Foram adicionadas ao questionário original questões referentes à opinião dos entrevistados a respeito de diversas políticas públicas do álcool. Ao todo, 16 questões específicas foram incluídas, divididas em seis grupos:

1) Políticas de acesso a bebidas alcoólicas: apoio às restrições de locais, quantidade e horários para a venda de bebidas alcoólicas; opinião sobre a ampliação da fiscalização dos pontos de venda.

2) Beber e dirigir: opiniões relacionadas às punições necessárias 


\section{ARTIGO ORIGINAL}

\section{CAROLINA DE MENESES GAYA}

CLARICE SANDI MADRUGA

ANDRÉ DE QUEIROZ CONSTANTINO MIGUEL

SANDRO MITSUHIRO

ILANA PINSKY

RAUL CAETANO

RONALDO LARANJEIRA

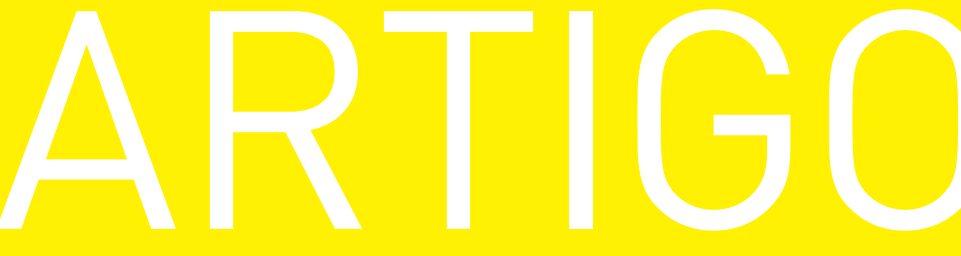

para quem é pego dirigindo alcoolizado (pessoas que ingeriram três ou mais doses).

3) Tratamento: opiniões referentes à ampliação dos programas de prevenção e tratamento para os transtornos relacionados ao consumo de álcool.

4) Impostos sobre bebidas: opiniões relacionadas à política de aumento dos impostos sobre as bebidas alcoólicas.

5) Campanhas de prevenção: ampliação das campanhas publicitárias de alerta aos riscos de beber; ampliação das mensagens de alerta nas propagandas e nos rótulos de bebidas; e maior desenvolvimento dos programas de prevenção sobre os riscos de beber.

6) Promoção e propagandas de bebidas: proibição das campanhas publicitárias de bebidas alcoólicas realizadas pelos fabricantes.

A partir dessas questões, foi instituída uma escala com respostas variando entre a aceitação completa de todas as políticas e a não aceitação de nenhuma das políticas mencionadas. $\bigcirc$ ponto de corte 6 foi eleito para definir o grupo dos menos favoráveis, referente aos $5 \%$ da população que menos aprova as políticas de álcool vigentes e/ou não apoia possíveis mudanças restritivas. A partir desse procedimento, foi criada uma variável binária para indicar o índice de não aceitação das políticas do álcool.

\section{Tratamento dos dados}

As análises estatísticas foram realizadas utilizando o software STATA versão 10. Devido aos múltiplos estágios do desenho amostral estratificado, todas as análises foram ponderadas em função das diferentes probabilidades de seleção (nas etapas) e de não resposta usando pós-estratificação. Todas as estimativas de prevalência e associação foram realizadas utilizando os comandos de pesquisa apropriados do STATA para gerar desvios padrão robustos. Odds ratios (OR) mutuamente ajustados com intervalos de confiança de 95\% (IC95\%) foram calculados para verificar as associações entre as características sociodemográficas e a opinião dos entrevistados sobre as políticas públicas.

\section{Aspectos éticos}

Este estudo foi aprovado pelo Comitê de Ética em Pesquisa da Universidade Federal de São Paulo (processo no 1671). Todos os participantes assinaram termo de consentimento livre e esclarecido.

\section{Resultados}

Caracterização da amostra

A amostra era constituída por $48 \%$ de homens e $52 \%$ de mulheres, com idade média \pm desvio padrão de $36 \pm 18$ anos; $55 \%$ dos entrevistados eram casados ou tinham relacionamentos estáveis. Verificou-se que 34\% da população concluiu o ensino fundamental, $38 \%$ terminou o ensino médio, e $28 \%$ possuía algum curso superior ou profissionalizante. Somente $37 \%$ da amostra total vivia em áreas urbanas.

Observou-se que 19\% dos avaliados eram fumantes, $57 \%$ eram usuários de álcool, e $4 \%$ haviam usado algum tipo de substância ilícita durante a vida.

\section{Apoio da população às políticas públicas}

Verificamos que entre 75 e $95 \%$ da população avaliada apoiava as três políticas apresentadas de restrição ao acesso a bebidas alcoólicas (Tabela 1). Nessa avaliação, de maneira geral, as mulheres se mostraram mais favoráveis que os homens. A proporção da amostra que apoiava as medidas era um pouco maior entre os idosos, indivíduos com menor escolaridade (até o ensino fundamental) e com renda mais baixa. Uma exceção a essa tendência foi a proporção que apoiava o aumento da idade para a compra de bebida alcoólica. Para esta política, o maior apoio estava entre as pessoas com renda maior. Com relação à região do país, o Norte e o Centro-Oeste apresentaram uma maior porcentagem de apoio às medidas de restrição quando comparadas às demais regiões.

A maior parte da amostra mostrou-se favorável às medidas de punição apresentadas para quem é advertido dirigindo alcoolizado. Destaca-se que 83,88\% do grupo de menores de 19 anos aprovaram tais medidas.

aumento dos impostos sobre as bebidas alcoólicas foi aprovado por quase $80 \%$ da população, sendo os participantes de maior idade os mais favoráveis a essa medida (83\%).

Entre todas as políticas, as referentes à ampliação dos programas de prevenção e tratamento foram as mais aprovadas pela população; já a restrição de campanhas publicitárias de bebidas recebeu o menor apoio, entre 54 e $66 \%$.

Observou-se que $86,2 \%$ dos indivíduos da amostra tiveram acesso a algum tipo de campanha publicitária de bebidas alcoólicas, sendo os maiores índices encontrados nas regiões Sul e Sudeste (90 e 91\%, respectivamente). Por outro lado, apenas 23,5\% da população teve acesso a campanhas de prevenção; nessa avaliação, a região Nordeste apresentou o menor índice de acesso, de 18,2\% (Tabela 2). 


\section{CAROLINA DE MENESES GAYA', CLARICE SANDI MADRUGA², ANDRÉ DE QUEIROZ CONSTANTINO MIGUEL ${ }^{2}$, SANDRO MITSUHIRO², ILANA PINSKY ${ }^{2}$, RAUL CAETANO ${ }^{3}$, \\ RONALDO LARANJEIRA ${ }^{2}$}

1 Instituto Nacional de Ciência e Tecnologia para Políticas Públicas do Álcool e Outras Drogas, Instituto de Psiquiatria, Universidade Federal de São Paulo (UNIFESP), São Paulo, SP. Universidade de Franca, Franca, SP. ${ }^{2}$ Universidade de Franca, Franca, SP. ${ }^{3}$ University of Texas School of Public Health, Dallas Regional Campus, Dallas, TX, EUA.

Tabela 1 - Prevalência de apoio às políticas de restrição ao uso e venda de bebidas alcoólicas, n (\%), parte 1

\begin{tabular}{|c|c|c|c|c|c|c|c|c|c|}
\hline & \multicolumn{4}{|c|}{ 1. Restrição ao acesso a bebidas alcoólicas } & \multicolumn{3}{|c|}{ 2. Beber $\mathrm{x}$ dirigir } & \multicolumn{2}{|c|}{ 3. Tratamento } \\
\hline & $\begin{array}{c}\text { Proibir } \\
\text { venda em } \\
\text { padarias, etc. }\end{array}$ & $\begin{array}{l}\text { Aumento da } \\
\text { fiscalização }\end{array}$ & $\begin{array}{l}\text { Restrição de } \\
\text { horário para } \\
\text { venda }\end{array}$ & $\begin{array}{l}\text { Aumento da } \\
\text { idade mínima } \\
\text { para compra }\end{array}$ & $\begin{array}{l}\text { Condenação } \\
\text { à prisão }\end{array}$ & $\begin{array}{l}\text { Suspensão } \\
\text { da CNH }\end{array}$ & Pagar multas & $\begin{array}{c}\text { Ampliar } \\
\text { programas de } \\
\text { tratamento }\end{array}$ & $\begin{array}{c}\text { Ampliar } \\
\text { programas de } \\
\text { prevenção }\end{array}$ \\
\hline \multicolumn{10}{|l|}{ Sexo } \\
\hline Masculino & $889(66.0)$ & $1.195(92.7)$ & $939(69.6)$ & $578(87.5)$ & 779 (59.6) & 997 (78.7) & $1.172(91.1)$ & $1.145(96.6)$ & $1.214(93.8)$ \\
\hline Feminino & $1.374(81.1)$ & $1.662(96.3)$ & $1.416(81.9)$ & $1.031(92.4)$ & $1.128(65.4)$ & $1.395(81.7)$ & $1.613(93.9)$ & $1.496(97.9)$ & $1.678(97.5)$ \\
\hline \multicolumn{10}{|l|}{ Idade } \\
\hline 14 a 19 anos & $538(71.2)$ & $702(91.3)$ & $550(70.1)$ & $318(83.8)$ & $476(62.5)$ & $569(73.2)$ & $706(92.2)$ & $681(98.5)$ & $733(95.6)$ \\
\hline 20 a 54 anos & $1.390(72.8)$ & $1.768(95.0)$ & $1.458(75.9)$ & $1.041(92.5)$ & $1.165(61.5)$ & $1.492(81.0)$ & $1.707(92.3)$ & $1.690(97.2)$ & $1.172(95.6)$ \\
\hline 55 ou mais & $335(82.8)$ & $387(96.5)$ & $347(84.3)$ & $250(85.8)$ & $266(68.7)$ & $331(85.3)$ & $372(94.5)$ & $370(95.9)$ & $387(96.5)$ \\
\hline \multicolumn{10}{|l|}{ Educação } \\
\hline Ensino fundamental & $825(0.6)$ & $974(95.6)$ & $880(85.1)$ & $635(91.0)$ & $692(70.1)$ & $842(84.1)$ & $944(93.9)$ & $931(96.9)$ & $983(96.8)$ \\
\hline Ensino médio & $964(73.8)$ & $1.232(94.2)$ & $996(77.0)$ & $648(90.3)$ & $816(60.9)$ & $1.032(80.1)$ & $1.217(93.2)$ & $1.182(97.9)$ & $1.257(95.4)$ \\
\hline Ensino superior ou mais & $474(65.8)$ & $651(93.9)$ & $479(63.7)$ & $326(89.3)$ & $399(55.8)$ & $518(75.9)$ & $624(90.0)$ & $628(96.8)$ & $652(94.8)$ \\
\hline \multicolumn{10}{|l|}{ Renda } \\
\hline Até $1 \mathrm{SM}$ & $220(79.7)$ & $264(95.6)$ & $232(82.9)$ & $156(89.6)$ & $190(67.7)$ & $213(78.7)$ & $254(93.4)$ & $246(95.1)$ & $267(97.2)$ \\
\hline 1 a $2 \mathrm{SM}$ & $278(78.1)$ & $335(93.1)$ & $289(82.6)$ & $192(89.6)$ & $209(58.2)$ & $261(73.7)$ & $324(90.3)$ & $333(98.2)$ & $337(94.6)$ \\
\hline 2 a $3 \mathrm{SM}$ & $133(73.3)$ & $174(96.1)$ & $146(79.0)$ & 87 (91.9) & $116(63.5)$ & $151(84.9)$ & $175(94.8)$ & $170(96.6)$ & $182(99.2)$ \\
\hline $4 \mathrm{SM}$ ou mais & $217(67.3)$ & $293(95.3)$ & $205(62.6)$ & $138(94.0)$ & $177(59.3)$ & $239(80.2)$ & $277(90.5)$ & $280(96.4)$ & $297(97.0)$ \\
\hline \multicolumn{10}{|l|}{ Região } \\
\hline $\mathrm{N} / \mathrm{CO}$ & 341 (78.6) & $424(4.8)$ & $369(81.7)$ & 255 (94.5) & $311(69.1)$ & $363(81.0)$ & $421(94.5)$ & $420(7.9)$ & 433 (97.9) \\
\hline $\mathrm{NE}$ & $698(74.9)$ & $833(92.5)$ & $724(78.9)$ & $519(89.3)$ & $552(61.8)$ & $683(78.3)$ & 805 (91.6) & $819(97.9)$ & $859(97.4)$ \\
\hline SE & $93.9(74.7)$ & $1.211(95.1)$ & $961(73.8)$ & $667(90.8)$ & $785(60.9)$ & $1.030(81.3)$ & $1.193(93.6)$ & $1.146(97.3)$ & $1.224(95.4)$ \\
\hline $\mathrm{S}$ & $285(65.3)$ & 389 (96.5) & $301(72.1)$ & 168 (86.2) & $259(63.1)$ & $3.316(80.1)$ & $366(89.2)$ & $356(95.3)$ & $376(91.0)$ \\
\hline Total & $2.263(75.3)$ & $2.857(95.0)$ & $2.355(78.4)$ & $1.609(89.8)$ & $1.907(63.5)$ & $2.392(79.5)$ & $2.785(92.6)$ & $2.741(97.2)$ & $2.892(96.2)$ \\
\hline
\end{tabular}

$\mathrm{CNH}=$ Carteira Nacional de Habilitação; $\mathrm{SM}=$ salário mínimo; $\mathrm{N}=$ Norte $\mathrm{CO}=$ Centro-Oeste; $\mathrm{NE}=$ Nordeste; $\mathrm{SE}=\mathrm{Sudeste}$.

Tabela 1 (continuação) - Prevalência de apoio às políticas de restrição ao uso e venda de bebidas alcoólicas, n (\%), parte 2

\begin{tabular}{|c|c|c|c|c|c|c|c|}
\hline & \multicolumn{2}{|c|}{ 4. Impostos } & \multicolumn{3}{|c|}{ 5. Campanhas de prevenção } & \multicolumn{2}{|c|}{ 6. Campanhas publicitárias de bebidas } \\
\hline & $\begin{array}{l}\text { Aumento dos } \\
\text { impostos }\end{array}$ & $\begin{array}{c}\text { Aumento das } \\
\text { campanhas de } \\
\text { alerta }\end{array}$ & $\begin{array}{c}\text { Ampliar a } \\
\text { prevenção nas } \\
\text { escolas }\end{array}$ & $\begin{array}{l}\text { Mensagens em } \\
\text { rótulos de } \\
\text { bebidas }\end{array}$ & $\begin{array}{c}\text { Mensagens de } \\
\text { alerta em } \\
\text { propagandas }\end{array}$ & $\begin{array}{c}\text { Proibir } \\
\text { propagandas }\end{array}$ & $\begin{array}{c}\text { Proibir o patrocínio } \\
\text { de eventos sociais }\end{array}$ \\
\hline \multicolumn{8}{|l|}{ Sexo } \\
\hline Masculino & $648(74.7)$ & $1.066(96.2)$ & $1.147(96.7)$ & $1.187(90.4)$ & $1.192(91.4)$ & $816(62.9)$ & $612(46.7)$ \\
\hline Feminino & $993(78.0)$ & $1.493(96.6)$ & $1.595(97.5)$ & $1.646(95.6)$ & $1.637(95.6)$ & $1.169(70.2)$ & $1.011(60.8)$ \\
\hline \multicolumn{8}{|l|}{ Idade } \\
\hline 14 a 19 anos & $337(67.5)$ & $617(93.7)$ & $670(95.9)$ & $718(92.1)$ & $704(91.5)$ & $421(55.9)$ & $322(44.5)$ \\
\hline 20 a 54 anos & $1.029(77.0)$ & $1.586(97.0)$ & $1.698(97.5)$ & $1.742(93.3)$ & $1.738(93.8)$ & $1.240(66.5)$ & $1.023(53.4)$ \\
\hline 55 ou mais & $275(83.1)$ & $356(96.3)$ & $374(96.5)$ & $373(93.4)$ & $377(95.0)$ & $325(81.0)$ & $278(69.3)$ \\
\hline \multicolumn{8}{|l|}{ Educação } \\
\hline Ensino fundamental & $603(78.3)$ & $885(95.4)$ & $920(96.0)$ & 963 (94.6) & $951(94.7)$ & 779 (77.3) & $642(63.7)$ \\
\hline Ensino médio & $657(73.0)$ & $1.084(96.8)$ & $1.179(96.8)$ & $1.236(93.6)$ & $1.221(92.9)$ & 777 (60.6) & $632(49.5)$ \\
\hline Ensino superior ou mais & $381(79.0)$ & $590(97.1)$ & $643(98.8)$ & $634(90.7)$ & $647(93.3)$ & 429 (61.9) & $349(48.6)$ \\
\hline \multicolumn{8}{|l|}{ Renda } \\
\hline Até $1 \mathrm{SM}$ & $149(73.3)$ & $228(93.4)$ & $238(94.6)$ & $265(95.2)$ & $255(93.1)$ & $178(62.6)$ & $148(52.6)$ \\
\hline 1 a 2 SM & $181(71.1)$ & 322 (98.9) & $335(98.3)$ & 343 (96.6) & $335(93.5)$ & $230(62.9)$ & $176(49.2)$ \\
\hline 2 a $3 \mathrm{SM}$ & $111(81.1)$ & $157(97.5)$ & $169(98.1)$ & $178(96.6)$ & $170(93.8)$ & $116(66.6)$ & $88(50.0)$ \\
\hline $4 \mathrm{SM}$ ou mais & $164(78.0)$ & $270(98.1)$ & $287(98.0)$ & $286(90.5)$ & $291(94.6)$ & $191(63.7)$ & $150(45.8)$ \\
\hline \multicolumn{8}{|l|}{ Região } \\
\hline $\mathrm{N} / \mathrm{CO}$ & $252(76.2)$ & $393(98.2)$ & $421(98.0)$ & $420(92.7)$ & $429(96.2)$ & $298(67.7)$ & $241(54.4)$ \\
\hline $\mathrm{NE}$ & $465(76.0)$ & $760(95.6)$ & $798(97.4)$ & $844(94.0)$ & $817(92.2)$ & $562(62.4)$ & $434(49.3)$ \\
\hline $\mathrm{SE}$ & 724 (79.7) & $1.072(96.4)$ & $1.153(96.8)$ & $1.202(93.9)$ & $1.195(93.7)$ & $855(69.5)$ & $730(57.9)$ \\
\hline $\mathrm{S}$ & $200(68.1)$ & 334 (96.3) & $370(96.8)$ & 367 (89.8) & 378 (93.3) & $270(65.2)$ & $218(50.8)$ \\
\hline Total & $1.641(76.6)$ & $2.559(96.2)$ & $2.742(96.6)$ & $2.833(94.2)$ & $2.819(93.7)$ & $1.985(66.0)$ & $1.623(54.0)$ \\
\hline
\end{tabular}

$\mathrm{SM}=$ salário mínimo; $\mathrm{N}=$ Norte $; \mathrm{CO}=$ Centro-Oeste $; \mathrm{NE}=$ Nordeste $; \mathrm{SE}=$ Sudeste . 


\section{ARTIGO ORIGINAL}

\section{CAROLINA DE MENESES GAYA}

CLARICE SANDI MADRUGA

ANDRÉ DE QUEIROZ CONSTANTINO MIGUEL

SANDRO MITSUHIRO

ILANA PINSKY

RAUL CAETANO

RONALDO LARANJEIRA

Tabela 2 - Fatores associados com a posição desfavorável às políticas públicas de restrição ao consumo do álcool

\begin{tabular}{lc}
\hline Variável & OR (IC95\%) \\
\hline Variáveis sociodemográficas & \\
Sexo & 1.00 \\
$\quad$ Feminino & $2.80(1.81-4.32)^{*}$ \\
$\quad$ Masculino & $0.97(0.95-0.98)^{\dagger}$ \\
Idade (constante) & 1.00 \\
Estado civil & $0.62(0.39-0.98)^{\ddagger}$ \\
$\quad$ Solteiro/separado & \\
$\quad$ Casado/relacionamento estável & 1.00 \\
Educação & $1.99(1.17-3.37)^{*}$ \\
$\quad$ Ensino fundamental & $3.36(1.95-5.77)^{*}$ \\
$\quad$ Ensino médio & $1.35(0.83-2.21)$ \\
$\quad$ Superior ou profissionalizante & $1.00(0.99-1.00)$ \\
Trabalhando & $1.81(0.99-3.29)$ \\
Renda familiar (constante) & \\
Vive em região metropolitana & $2.50(1.09-5.72)^{*}$ \\
Uso de substâncias & $2.22(1.21-4.06)^{*}$ \\
Abuso/dependência de álcool & $1.53(1.15-2.03)^{*}$ \\
Uso de tabaco & \\
Uso de drogas & $1.07(0.57-2.01)$ \\
Outros & $0.79(0.44-1.39)$ \\
Problema com uso de outras pessoas & $0.79(0.43-1.43)$ \\
Acesso a propagandas & $0.41(0.24-0.69)^{*}$ \\
Acesso a programas de prevenção & \\
Religiosidade & \\
\hline
\end{tabular}

IC95\% = intervalo de confiança de 95\%; OR = odds ratio.

* Positivamente associadas com posição desfavorável às políticas de restrição.

${ }^{\dagger}$ Associação inversa.

* Variável de referência.

Na avaliação do perfil dos participantes menos favoráveis às políticas relacionadas ao uso de álcool, os homens apresentaram quase três vezes mais chances de ser desfavoráveis do que as mulheres ( $O R=2.80, I C 95 \%=1.81-432)$. Observou-se, também, que o nível educacional esteva significativamente associado à posição desfavorável às políticas, numa relação dose-resposta (efeito trend).
Abuso e/ou dependência de álcool ( $\mathrm{OR}=2.50, \mathrm{IC} 95 \%=$ 1.09-5.72), uso de tabaco (OR $=2.22$, IC95\% $=1.21-4.06)$ e de outras drogas ( $\mathrm{OR}=1.53$, IC95\% $=1.15-2.03)$ mostraram-se significativamente associados com a desaprovação das medidas apresentadas. Observou-se ainda que não ter relacionamento estável e não possuir religiosidade foram fatores associados com posições mais desfavoráveis. 


\section{CAROLINA DE MENESES GAYA ${ }^{1}$, CLARICE SANDI MADRUGA², ANDRÉ DE QUEIROZ CONSTANTINO MIGUEL ${ }^{2}$, SANDRO MITSUHIRO ${ }^{2}$, ILANA PINSKY ${ }^{2}$, RAUL CAETANO ${ }^{3}$, \\ RONALDO LARANJEIRA ${ }^{2}$}

${ }^{1}$ Instituto Nacional de Ciência e Tecnologia para Políticas Públicas do Álcool e Outras Drogas, Instituto de Psiquiatria, Universidade Federal de São Paulo (UNIFESP), São Paulo, SP. Universidade de Franca, Franca, SP. ${ }^{2}$ Universidade de Franca, Franca, SP. ${ }^{3}$ University of Texas School of Public Health, Dallas Regional Campus, Dallas, TX, EUA.

\section{Discussão}

Esta pesquisa abordou iniciativas e políticas públicas relacionadas ao acesso, promoção, prevenção e tratamento dos problemas relacionados ao uso do álcool, que, na opinião dos entrevistados, deveriam ser aumentadas, reduzidas ou manter-se inalteradas. Além disso, foi avaliado o perfil dos participantes contrários às medidas restritivas ao consumo de álcool.

A grande maioria da população brasileira apoiou o aumento de programas preventivos ao uso do álcool nas escolas, programas de tratamento para o alcoolismo e campanhas governamentais de alerta sobre os riscos do álcool. Tal resultado vem ao encontro de estudos prévios e também a dados nacionais advindos do mesmo levantamento sobre motivações para o tratamento do alcoolismo 13,14 .

Mais da metade da população adulta (76,7\%) defendeu o aumento dos impostos sobre as bebidas alcoólicas. Convém ressaltar que, em diversos países, o aumento da taxação e do preço das bebidas alcoólicas resultou na diminuição do consumo e, consequentemente, dos problemas relacionados ao álcool $\left.\right|^{1,15-17}$.

O aumento da idade mínima de 18 anos para a venda de bebidas alcoólicas foi defendido por mais de três quartos da amostra pesquisada. Vale ressaltar que menos de 10\% dos entrevistados concordam com a redução da idade mínima para a venda de bebidas. Deve-se considerar que os EUA, há mais de 20 anos, adotaram a idade de 21 anos como limite mínimo para a venda e o consumo de bebidas alcoólicas ${ }^{18}$. Atualmente, não se contesta que a implementação e a fiscalização dessa medida foi um grande avanço na saúde dos jovens. Diversos estudos americanos evidenciaram os benefícios dessa prática, tais como diminuição dos acidentes de automóveis envolvendo jovens, queda da mortalidade e diminuição do consumo de álcool entre os jovens 1,19-22 $^{1}$

De acordo com o Estatuto da Criança e do Adolescente (artigo 243 da Lei 8.069/90), é motivo de detenção e multa "vender, fornecer ainda que gratuitamente, ministrar ou entregar de qualquer forma, a criança ou adolescente, sem justa causa, produtos cujos componentes possam causar dependência física ou psíquica". Caberia aos órgãos de fiscalização, sobretudo dos municípios, garantir a aplicação do Estatuto no sentido de coibir a venda de bebida alcoólica para menores. No Brasil, entretanto, esse dispositivo legal não tem sido cumprido, e, em muitas regiões, menores de 18 anos não encontram dificuldade alguma para comprar bebidas alcoólicas. A solução apontada pela maioria dos entrevistados foi o aumento da fiscalização do comércio em relação à venda de bebidas alcoólicas para menores.
Controlar as condições de venda ao consumidor é uma medida capaz de interferir na disponibilidade física e na acessibilidade às bebidas alcoólicas ${ }^{23,24}$. $\bigcirc$ objetivo, também neste caso, é reduzir os danos decorrentes da ingestão alcoólica. Nesse sentido, os estabelecimentos que mais influenciam o uso do álcool são aqueles que vendem bebidas para serem consumidas no próprio local. Para a maioria da amostra, os estabelecimentos não deveriam servir bebidas alcoólicas para clientes que já estão embriagados. Muitos participantes também concordam que padarias, confeitarias e mercearias deveriam ser proibidas de vender bebidas alcoólicas.

Quanto à restrição do horário de venda de bebidas alcoólicas, mais de $70 \%$ da população defende essa medida. Diversos municípios brasileiros têm adotado leis que regulamentam - horário de funcionamento dos estabelecimentos que comercializam bebidas alcoólicas. Estudos revelam que essa medida, conhecida como "Lei Seca", contribuiu para a redução dos homicídios e da violência doméstica ${ }^{6}$.

A União Europeia, em 1995, aprovou um documento conhecido como The European Charter on Alcohol, que estabelece alguns princípios e objetivos para promover e proteger a saúde e o bem-estar dos jovens. Essa declaração tem como objetivos: diminuir e postergar o consumo de álcool entre jovens; reduzir os danos diretos ou indiretos produzidos pelo consumo de bebidas alcoólicas; aumentar a educação e o envolvimento dos jovens nas políticas públicas relacionadas ao álcool; minimizar as pressões para que os jovens bebam, especialmente em relação às promoções e distribuição gratuita de álcool, propaganda e disponibilidade de bebidas em eventos sociais; favorecer ações contra a venda ilegal de álcool; garantir e/ou aumentar o acesso a serviços de saúde, especialmente para jovens com problemas pessoais ou familiares relacionados ao uso de álcool.

No Brasil, recentemente, ocorreu um avanço em relação às leis que restringem $\mathrm{O}$ uso de tabaco. $\bigcirc$ país foi pioneiro na implementação de campanhas antitabaco nos maços de cigarro ${ }^{25}$, na proibição de comerciais televisivos e do uso do cigarro em locais públicos. Tais iniciativas tiveram um impacto positivo, diminuindo a prevalência do uso de cigarros em diferentes faixas etárias ${ }^{26-28}$. Essas evidências, bem como as de estudos prévios mostrando os resultados de iniciativas semelhantes em outras nações, sugerem que a adoção de medidas restritivas tem impacto no controle do acesso, promoção, taxação e consumo do álcool e pode reduzir os problemas associados a essa droga em nosso país 10,11,16,29. A grande maioria dos respondentes apoiou medidas de restrição às propagandas, como por exemplo, exibir 


\section{ARTIGO ORIGINAL}

CAROLINA DE MENESES GAYA

CLARICE SANDI MADRUGA

ANDRÉ DE QUEIROZ CONSTANTINO MIGUEL

SANDRO MITSUHIRO

ILANA PINSKY

RAUL CAETANO

RONALDO LARANJEIRA

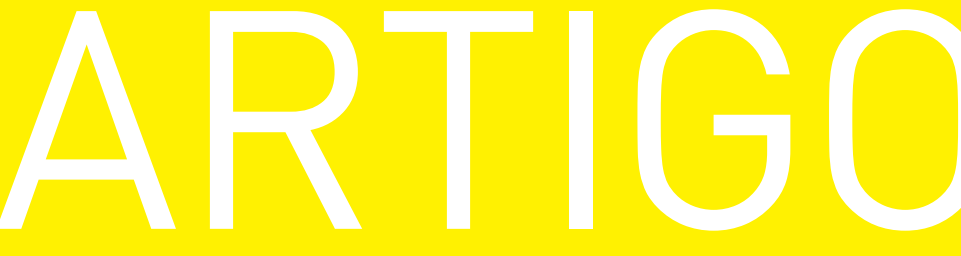

mensagens de alerta sobre os riscos e problemas causados pelo uso de bebidas alcoólicas, proibir propagandas de bebidas na televisão e proibir que fabricantes de bebidas patrocinem eventos culturais e esportivos.

Ainda que a grande maioria da população investigada tenha sido favorável às medidas sugeridas, verificou-se, neste estudo, que algumas características da população estiveram independentemente associadas à não aceitação das políticas restritivas de forma geral. Participantes do sexo masculino, sem relacionamento estável, sem religião, jovens, tabagistas, usuários de drogas ilegais e que apresentam abuso e/ou dependência de álcool tenderam a ser menos favoráveis a políticas restritivas. Esses achados estão de acordo com estudos prévios realizados em países desenvolvidos ${ }^{8,30,31}$. Surpreendentemente, maior nível educacional foi identificado como um fator significativamente associado com a não aceitação das políticas de restrição. Embora contraintuitiva, tal relação seguiu um padrão de dose-resposta explícito, onde os participantes com ensino médio completo tiveram duas vezes mais chances de se colocar contra as políticas propostas, e os participantes com ensino superior, três vezes mais chances de ser desfavoráveis às medidas. Tal evidência é, sem dúvida, preocupante e abre a questão de o quanto a educação formal não necessariamente leva à compreensão mais ampla da necessidade de medidas voltadas para o bem-estar coletivo, bem como do impacto do uso abusivo de álcool na sociedade. Preconizam-se iniciativas que esclareçam os benefícios de tais medidas para a população, focadas ao público menos favorável. Cabe ressaltar que as altas prevalências de aceitação, pela população, da grande maioria das políticas restritivas avaliadas abre espaço para a implementação de políticas restritivas de uso, acesso e propaganda do álcool como uma estratégia de manutenção da saúde pública.

\section{Conclusão}

Conclui-se que a vasta maioria da população apoia as políticas vigentes, assim como a implementação de novas leis de restrição ao consumo de álcool. As políticas de incentivo a tratamento e a ampliação de programas de prevenção e de restrição de acesso a bebidas alcoólicas receberam a maior adesão entre os indivíduos avaliados. Os prejuízos sociais relacionados ao consumo abusivo preconizam uma mudança na legislação, bem como um aumento da fiscalização das leis vigentes. Ações focadas para o público masculino, com maior escolaridade e para os usuários de álcool e demais substâncias psicotrópicas são fundamentais, uma vez que essa população se mostrou menos susceptível a tais mudanças. A compreensão e o apoio da população são imprescindíveis para a implementação bem-sucedida de políticas públicas relacionadas o uso de álcool.

\section{Agradecimentos}

Este estudo foi financiado pela Secretaria Nacional Antidrogas (SENAD) e pelo Conselho Nacional de Desenvolvimento Científico e Tecnológico (CNPq) durante - desenho e a realização da pesquisa, e pela Coordenação de Aperfeiçoamento de Pessoal de Nível Superior (CAPES) durante as fases de análise e interpretação dos dados.

\section{Correspondência:}

Carolina de Meneses Gaya

Rua Zacarias de Goís, 715/191A, Campo Belo

04610-001 - São Paulo, SP

Tel.: (11) 8252.2305

E-mail: carolmgaya@yahoo.com.br, carolinagaya@usp.br

Financiamento: Secretaria Nacional Antidrogas (SENAD), Conselho Nacional de Desenvolvimento Científico e Tecnológico (CNPq) e Coordenação de Aperfeiçoamento de Pessoal de Nível Superior (CAPES).

Conflitos de interesse inexistentes.

\section{Referências}

1. Rehm J, Mathers C, Popova S, Thavorncharoensap M, Teerawattananon Y, Patra J. Global burden of disease and injury and economic cost attributable to alcohol use and alcohol-use disorders. Lancet. 2009;373:222333.

2. Caetano R, Madruga C, Pinsky I, Laranjeira R. Drinking patterns and associated problems in Brazil. Adicciones. 2013;25:287-93.

3. World Health Organization (WHO). Global strategy to reduce harmful use of alcohol. Switzerland: WHO; 2010.

4. Caetano R, Laranjeira R. A 'perfect storm' in developing countries: economic growth and the alcohol industry. Addiction. 2006;101:149-52.

5. Melotti R, Heron J, Hickman M, Macleod J, Araya R, Lewis $G$, et al. Adolescent alcohol and tobacco use and early socioeconomic position: the ALSPAC birth cohort. Pediatrics. 2011;127:e948-55.

6. Duailibi S, Ponicki W, Grube J, Pinsky I, Laranjeira 


\section{CAROLINA DE MENESES GAYA', CLARICE SANDI MADRUGA², ANDRÉ DE QUEIROZ CONSTANTINO MIGUEL ${ }^{2}$, SANDRO MITSUHIRO ${ }^{2}$, ILANA PINSKY ${ }^{2}$, RAUL CAETANO ${ }^{3}$, \\ RONALDO LARANJEIRA ${ }^{2}$}

${ }^{1}$ Instituto Nacional de Ciência e Tecnologia para Políticas Públicas do Álcool e Outras Drogas, Instituto de Psiquiatria, Universidade Federal de São Paulo (UNIFESP), São Paulo, SP. Universidade de Franca, Franca, SP. ${ }^{2}$ Universidade de Franca, Franca, SP. ${ }^{3}$ University of Texas School of Public Health, Dallas Regional Campus, Dallas, TX, EUA.

R, Raw $M$. The effect of restricting opening hours on alcohol-related violence. Am J Public Health. 2007;97:2276-80.

7. Huckle T, Pledger M, Casswell S. Increases in typical quantities consumed and alcohol-related problems during a decade of liberalizing alcohol policy. I Stud Alcohol Drugs. 2011:73:53-62.

8. Babor TF, Caetano R. Evidence-based alcohol policy in the Americas: strengths, weaknesses, and future challenges. Rev Panam Salud Publica. 2005;18:327-37.

9. Mart SM. Top priorities for alcohol regulators in the United States: protecting public health or the alcohol industry? Addiction. 2012;107:259-62.

10. Bosque-Prous M, Espelt A, Guitart AM, Bartroli M, Villalbí JR, Brugal MT. Association between stricter alcohol advertising regulations and lower hazardous drinking across European countries. Addiction. 2014;109:1634:43.

11. Nelson JP. Alcohol affordability and alcohol demand: cross-country trends and panel data estimates, 1975 to 2008. Alcohol Clin Exp Res. 2014;38:1167-75.

12. Caetano R, Vaeth PA, Ramisetty-Mikler S, Rodriguez LA. Hispanic Americans Baseline Alcohol Survey: alcoholic beverage preference across Hispanic national groups. Alcohol Clin Exp Res. 2009;33:150-9.

13. Cook WK, Bond J, Greenfield TK. Are alcohol policies associated with alcohol consumption in low-and middle-income countries? Addiction. 2014;109:108190.

14. Madruga CS, De Saibro P, Ferri CP, Caetano R, Laranjeira R, Pinsky I. Correlated factors and prevalence of alcohol treatment in Brazil: a national survey. Addict Disord Their Treat. 2014;15:01.

15. Ataguba JE. Alcohol policy and taxation in South Africa: an examination of the economic burden of alcohol tax. Appl Health Econ Health Policy. 2011;10:65-76.

16. Casswell S, Huckle T, Wall M, Yeh LC. International alcohol control study: pricing data and hours of purchase predict heavier drinking. Alcohol Clin Exp Res. 2014;38:1425-31.

17. Nelson JP. Estimating the price elasticity of beer: metaanalysis of data with heterogeneity, dependence, and publication bias. J Health Econ. 2014;33:180-7.

18. WHO. Global Status Report on Alcohol 2004. Geneva: WHO; 2004.
19. Rehm J, Taylor B, Room R. Global burden of disease from alcohol, illicit drugs and tobacco. Drug Alcohol Rev. 2006;25:503-13.

20. Paschall MJ, Grube JW, Kypri K. Alcohol control policies and alcohol consumption by youth: a multinational study. Addiction. 2009;104:1849-55.

21. Navarro HJ, Doran CM, Shakeshaft AP. Measuring costs of alcohol harm to others: A review of the literature. Drug Alcohol Depend. 2011;114:87-99.

22. Catalano RF, Fagan AA, Gavin LE, Greenberg MT, Irwin CE Jr, Ross DA, et al. Worldwide application of prevention science in adolescent health. Lancet. 2012;379:1653-64.

23. Caetano R, Mills B, Pinsky I, Zaleski M, Laranjeira $\mathrm{R}$. The distribution of alcohol consumption and the prevention paradox in Brazil. Addiction. 2012;107:608.

24. Mckee PA, Nelson TF, Toomey TL, Shimotsu ST, Hannan PJ, Jones-Webb RJ. Adopting local alcohol policies: a case study of community efforts to regulate malt liquor sales. Am J Health Promot. 2012;26:e8694.

25. Instituto Nacional de Cancer (INCA). Brazil: health warnings on tobacco products 2009 [Internet]. 2009 [cited 2010 Feb 10]. http://www.tobaccolabels.ca/wp/ wp-content/uploads/2013/12/Brazil-2008-HealthWarnings-on-Tobacco-Products-Report-English.pdf

26. Rodrigues MC, Viegas CA, Gomes EL, Morais JP, Zakir JC. Prevalence of smoking and its association with the use of other drugs among students in the Federal District of Brasilia, Brazil. J Bras Pneumol. 2009;35:986-91.

27. Szklo AS, de Almeida LM, Figueiredo VC, Autran M, Malta D, Caixeta R, et al. A snapshot of the striking decrease in cigarette smoking prevalence in Brazil between 1989 and 2008. Prev Med. 2012;54:162-7.

28. Gigliotti A, Figueiredo VC, Madruga CS, Marques AC, Pinsky I, Caetano R, et al. How smokers may react to cigarette taxes and price increases in Brazil: data from a national survey. BMC Public Health. 2014;14:327.

29. Healey C, Rahman A, Faizal M, Kinderman P. Underage drinking in the UK: changing trends, impact and interventions. A rapid evidence synthesis. Int J Drug Policy. 2014:25:124-32.

30. Pinsky I, Sanches M, Zaleski M, Laranjeira R, Caetano 


\section{ARTIGO ORIGINAL}

CAROLINA DE MENESES GAYA

CLARICE SANDI MADRUGA

ANDRÉ DE QUEIROZ CONSTANTINO MIGUEL

SANDRO MITSUHIRO

ILANA PINSKY

RAUL CAETANO

RONALDO LARANJEIRA

R. Opinions about alcohol control policies among Brazilians: the first national alcohol survey. Contemp Drug Probl. 2007;34:635-47.

31. Allamani A, Voller F, Decarli A, Casotto V, Pantzer K, Anderson P, et al. Contextual determinants of alcohol consumption changes and preventive alcohol policies: a 12-country European study in progress. Subst Use Misuse. 2011;46:1288-303.

\section{PARTICIPE DA ABP TAMBÉM PELA INTERNET.}

Conheça os canais de comunicação da sua associaçāo e compartilhe as informações sobre psiquiatria.

I) /facebook

in Associação Brasileira de Psiquiatria

You

đưbe

/canaldapsiquiatria

@abpbrasil

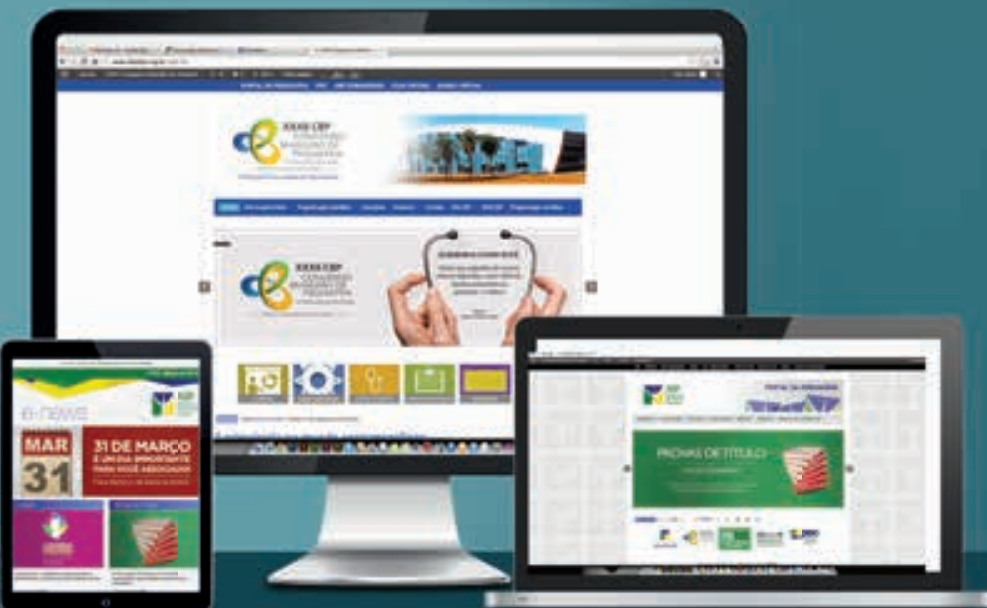

